\section{Cerebrovascular Diseases}

\section{Abstracts}

Cerebrovasc Dis 2006;22:318-329

DOI: $10.1159 / 000094832$

The abstracts are only available online, free of charge, under www.karger.com/doi/10.1159/000094832

\title{
Second International Stroke Summit
}

Nanjing, China, August 17-21, 2006

\section{Selected Abstracts}

Chairpersons

Julien Bogousslavsky, Lausanne, Switzerland

Xinfeng Liu, Nanjing, China

Chuanzhen Lv, Shanghai, China

Xueming Yi, Nanjing, China

Kangning Zhang, Nanjing, China

Shakir Husian, New Delhi, India

Luxiang Chi, Chongqing, China

Chuanqiang $P u$, Beijing, China

Gang Zhao, Xi'an, China

Zuohan Li, Nanjing, China

Xinsheng Ding, Nanjing, China

Yingdong Zhang, Nanjing, China

Ming Liu, Chengdu, China

Jie Shuai, Chongqing, China

Jixin Shi, Nanjing, China

Guangming Lu, Nanjing, China

Shisen Jiang, Nanjing, China

Zhenguo Liu, Twin Cities, Minn., USA

Jame Wang, Knoxville, Tenn., USA

Geoffrey Donnan, Heidelberg, Vic., Australia

Werner Hacke, Heidelberg, Germany

Veronica Skvortsova, Moscow, Russia

Gelin Xu, Nanjing, China 


\section{$\mathrm{NA}^{+}, \mathrm{K}^{+}$-ATPase Activity in Apoptotic Neurons and Regulation by Src Tyrosine Kinases}

\author{
SP. YU \\ Department of Pharmaceutical Science, Medical \\ University of South Carolina, Charleston, SC, USA
}

Objective: The ATP level and $\mathrm{Na}^{+}, \mathrm{K}^{+}$-ATPase activity are impaired in ischemic stroke. The present work is to understand the regulation and consequences of these pathological changes in central neurons. Methods: The $\mathrm{Na}^{+}, \mathrm{K}^{+}$-ATPase activity was measured in cultured mouse cortical neurons as the whole-cell currents sensitive to the $\mathrm{Na}^{+}, \mathrm{K}^{+}$-ATPase inhibitor ouabain or strophanthidin. Apoptotic cell death was induced by serum deprivation and staurosporine. Results: $\mathrm{The} \mathrm{Na}^{+}$-pump current was markedly suppressed several hours after the onset of an insult. The dysfunction of the $\mathrm{Na}^{+}$pump was accompanied with a marked depletion of intracellular $\mathrm{K}^{+}$, an event has been identified as an essential apoptotic mechanism linking to cell shrinkage, caspase activation, DNA damage, and cytochrome $\mathrm{c}$ release. The $\mathrm{Na}^{+}-$ pump dysfunction was mediated by a significant $(\sim 50 \%)$ reduction in cellular ATP level, changes in ATP/ADP ratio, and increased reactive oxygen species. Pyruvate and succinate prevented ATP deficiency, ROS increase, and cell death. The $\mathrm{Na}^{+}$-pump current was blocked by tyrosine kinase inhibitors. Western blot showed lower tyrosine phosphorylation levels of the pump $\alpha 3$ subunit in apoptotic cells. Intracellular injection of anti-lyn antibody gradually blocked the pump current, and the current was enhanced by the active lyn kinase. Additional evidence suggests that lyn and the $\alpha 3$ subunit can form a protein-protein complex. Signifying the importance of $\mathrm{Na}^{+}, \mathrm{K}^{+}$-ATPase in apoptosis, ouabain at a low concentration $(0.1 \mu \mathrm{M})$ drastically sensitized the neuron susceptibility to apoptotic insults. Conclusion: It is suggested that a certain but noticeable decrease of ATP production and impaired $\mathrm{Na}^{+}$-pump activity play imperative roles in cellular $\mathrm{K}^{+}$depletion and apoptosis. The decreased $\mathrm{Na}^{+}$-pump function was likely mediated by reduced tyrosine kinase phosphorylation.

\section{Effect of Noninvasive Intranasal Delivery Nerve Growth Factor on the Migration and Differentiation of SVZ Stem Cells in Sarin-Poisioned Rats}

\section{W. Zhu, X. Fan, G. Xu, M. Ma, R. Zhang, X. Liu \\ Department of Neurology, Jinling Hospital, Nanjing University School of Medicine, Nanjing, China}

Background and purpose: Sarin, an organophosphorus compound, can induce neuronal cells loss significantly and result in longterm neurological deficits. It was confirmed that enhanced proliferation of neural progenitors occurred in the subventricular zone (SVZ) of the brain following organophosphorus compounds. NGF is an active polypeptide with potent multipotential trophic effects and can induce the migration and differentiation of stem cell in vitro. This study was aimed to investigate the effect of NGF intranasal administration on the SVZ stem cells in sarin-exposured rats. Methods: Sprague-Dawley rats were treated with $2 \mathrm{LD}_{50}$ of sarin (intramuscular injection) and administered by peritoneal injection of atropine sulphate, pralidoxime 1 min after intoxication. NGF or saline was intranasal administered twice a day, from post-sarin days 1-4. Bromodeoxyurdidine (BrdU), which was used to label replicating neural progenitors, was injected $50 \mathrm{mg} / \mathrm{kg}$ thrice daily, from post-sarin days $2-4$. Rats were sacrificed on post-sarin day $5,7,14,21$ or 28 . The migration and differentiation of SVZ stem cells were labeled and quantified by BrdU/GFAP and $\mathrm{BrdU} / \mathrm{NeuN}$ double staining on brain sections of damaged brain regions (CA1 hippocampal layer and amygdala). Results: On post-sarin day 5 and 7, the number of BrdU positive cells labeled by immunochemistry increased significantly in SVZ both in the rats with IN NGF and saline, while not in the CA1 hippocampal layer and amygdala. IN NGF seemed to promote the migration and differentiation of the replicating neural stem cells from SVZ towards damaged regions. On post-sarin day 28, IN NGF increased the number of BrdU-labeled cells in the CA1 hippocampal layer and amygdala, compared to rats with IN saline. Interestingly, IN NGF promoted the differetiation of BrdU-positive cells into new neurons by BrdU/GFAP or BrdU/NeuN double staining. Conclusions: This results indicate NGF can enter the brain by intranasal pathway, promote the migration of neonatal stem cells from SVZ towards damaged regions and the differentiation of stem cells into new neurons in sarin-exposure rats. Noninvasive intranasal delivery NGF should be a potential treatment to promote the neural regeneration and repair process in the brain of sarin-poisoned rats.

\section{Neurovascular Remodeling after Cerebral Ischemic Stroke}

\author{
L. Wei \\ Department of Pathology, Medical University of \\ South Carolina, Charleston, SC, USA
}

Objective: Neurovascular unit repair and remodeling, which are largely dependent on angiogenesis and neurogenesis, is imperative for tissue repair and functional recovery after brain ischemia. Endogenous angiogenesis and neurogenesis after ischemic stroke have been investigated in animal models of focal cerebral ischemia. Method: To study neurovascular remodeling in the developing brain, P7 neonatal rats were anesthetized and selective branches of the right middle cerebral artery (MCA) were occluded to generate a whisker-barrel ischemic stroke. Replication-incompetent retroviral labeling was applied to mark neural stem cells of the subventricular zone (SVZ) by injection into the left lateral ventricle. Animals also received daily

\begin{tabular}{ll}
\hline KARGER & (c) 2006 S. Karger AG, Basel \\
Fax +4161306 1234 & 1015-9770/06/0224-0318 \\
E-Mail karger@karger.ch & Accessible online at: \\
www.karger.com & www.karger.com/ced
\end{tabular}


BrdU injections following stroke to label proliferating cells. Animals were sacrificed 1-5 weeks after ischemia, frozen sections were collected and immunostained for the retroviral marker, proliferation marker BrdU, neuronal marker NeuN, and others. Results: Following focal cerebral ischemia, neurogenesis and migration of cells increased compared with sham controls. Immunostaining revealed that 3 weeks after stroke, approximately $50 \%$ of all neurons in the bordering area were newly proliferated neurons derived from SVZ precursor cells, while $10-25 \%$ of neurons in contralateral side and other parts of hemisphere were identified as new cells. Erythropoietin (EPO) showed protective effect against ischemia-induced cell death and stimulation on endogenous angiogenesis and neurogenesis. EPO treatment increased STAT-5 and Bcl-2 levels, markedly attenuated apoptotic cell death and reduced ischemic infarct in the cortex. EPO increased BrdU/GLUT-1 and BrdU/NeuN positive cells after stroke, and increased the VEGF and Tie-2 expression. Conclusion: Our data support that the developing brain is highly plastic in response to ischemia, and the enhanced endogenous angiogenesis and neurogenesis may serve as a self-repair mechanism in the damaged brain.

\section{Olfactory Ensheathing Cells Conditioned Medium Protects against Glutamate-Induced Hippocampus Neurons Injury in vitro}

\author{
H. Zhang, X. Mao, G. Xu, X. Liu \\ Department of Neurology, Jinling Hospital, Nanjing \\ University School of Medicine, Nanjing, China
}

Background and purpose: The olfactory nerves have the unique property of being able to regenerate and restore normal functional connections throughout adult life. This capacity is thought to be due in part to olfactory ensheathing cells (OECs). Many studies in vivo and in vitro have demonstrated the potential of OECs for the repair of spinal cord injury by some mechanisms including long tract regeneration, axonal sparing, plasticity associated with novel polysynaptic pathways and neuroprotection of corticospinal tract neurons. Hippocampus Neurons damage induced by glutamate is the common ischemic cerebral injury model in vitro. This study investigates whether OECs and olfactory ensheathing cell conditioned medium (OECCM) could protect injured hippocampus neurons and demonstrates the possible mechanisms of neuroprotection. Methods: OECs were isolated from adult rat olfactory bulb and purified by a method based on the different rates of attachment of the various harvested cell types. Immunofluorescence tests were used to identify OECs. OECCM were collected and concentrated. The model of glutamate excitotoxicity on primary cultured hippocampus neurons from neonatal rats was set up. Hippocampus neurons were randomly divided into 4 groups: uninjured hippocampus neurons (group A), hippocampus neurons damaged by glutamate (group B), damaged hippocampus neurons cultured by OECCM (group C), injured hippocampus neurons cocultured by OECs (group D). The apoptosis were estimated by TUNEL staining and flow cytometer. The dynamic expression of nitric oxide synthase (NOS) was investigated with NADPH-diaphorase histochemical method. The number of survival hippocampus neurons and viability were tested by counting and MTT assays respectively. Morphology and neurite length of hippocampus neurons were observed under phase-contrast microscopy. Results:
Compared with group B, the survival rate of hippocampus neurons was remarkably increased and hippocampus neurite were longer in group $\mathrm{C}$ and group D. There was no statically difference between group $\mathrm{C}$ and group $\mathrm{D}$ in the survival rate and neurite length of hippocampus neurons. The decreasing of apoptosis cell and increasing of expression of NOS in group C were observed than group B. Conclusions: OECCM could protect hippocampus neurons against damages induced by glutamate, which has similar to direct cellular contact between OECs and injured neurons in neuroprotective effects. OECCM could decrease apoptosis neurons by increasing expression of NOS. It is suggested that OEC protect injured hippocampus neurons in vitro by secretion of neurotrophic factors.

\section{The Protective Effect of MK886 on Human Umbilical Vein Endothelial Cell Damaged by ox-LDL}

\author{
W. Zhu, G. Zhou, H. Zhang, X. Mao, X. Fan \\ Department of Neurology, Jinling Hospital, Nanjing \\ University School of Medicine, Nanjing, China
}

Objective: To investigate the role of CysLTs in the vitro model of human umbilical vein endothelial cell (HUVEC) damaged by oxidized low density lipoprotein (ox-LDL), and the protective effect by MK886 on this model. Methods: It was divided with 3 groups: HUVEC was cultured in vitro as group A. HUVEC was incubated with ox-LDL $(100 \mathrm{mg} / \mathrm{L})$ lasting for $24 \mathrm{~h}$ as group B. HUVEC was incubated with ox-LDL $(100 \mathrm{mg} / \mathrm{L})$ and MK886 $(20 \mu \mathrm{mol} / \mathrm{L})$ lasting for $24 \mathrm{~h}$ as group C. CysLTs (ELISA) and cell apoptosis (flowcytometry) examination were assessed in supernatant fluid among 3 groups. Results: The level of CysLTs in group B was higher than that in group A $(p<0.05)$, while CysLTs in group $C$ was lower than that in group B $(\mathrm{p}<0.05)$. The numbers of cell apoptosis in group A $(p<0.05)$ and group $C(p<0.01)$ were lower compared to that in group B. Conclusions: CysLTs plays a role in the vitro model of HUVEC damaged by ox-LDL, and is one of mechanisms in proceed of early atherosclerosis. MK886 can produce protective effect on HUVEC damaged by ox-LDL, which perhaps is a potential treatment for atherosclerosis.

\section{Intranasal Administration of Transforming Growth Factor $\beta 1$ Promotes Progenitor Cells Migration and Differentiation in Subventricular Zone following Focal Cerebral Ischemia}

\author{
M. Ma, G. Xu, W. Zhu, R. Zhang, Q. Yin \\ Department of Neurology, Jinling Hospital, Nanjing \\ University School of Medicine, Nanjing, China
}

Background: Progenitor cells in the subventricular zone (SVZ) can proliferate throughout the life of the adult brain. After focal cerebral ischemia, the progenitor cells can migrate into the lesions and differentiate into neurons. Transforming growth factor $\beta 1$ (TGF $\beta 1$ ) is not 
only an anti-inflammatory cytokine with neurogenic potential but also a key molecule involved in neural stem cells differentiation. This study investigated whether intranasal delivery of TGF $\beta 1$ would affect SVZ stem cell migration and differentiation after focal cerebral ischemia. Methods: After permanent right middle cerebral artery occlusion (MCAO), the mouse were divided randomly into 2 groups (MCAO + TGF $\beta 1$ group; MCAO group) according to with or without 2 days TGF $\beta 1$ intranasal administration. TGF $\beta 1$ was intranasal administrated at 24 and $48 \mathrm{~h}$ in adult mouse. Proliferating cells were labeled with 5-bromodeoxyuridine (BrdU) twice daily for three consecutive days from the day after ischemia. Mouse were killed at 7, 14, or 28 days after stroke. Immunohistochemistry for Brdu and neuron- or gliocytespecific markers were used to characterize neural stem cells and their progeny in SVZ. Results: In SVZ, doublecortin-immunoreactive cells migrated into the peri-infarct region of the striatrum after MCAO at days 5-14. At day 28, some of BrdU-labeled cells were immunopositive for the mature neuron-specific marker NeuN, but a few were co-labeled with gliocyte-specific markers. After intranasal administration of TGF $\beta 1$, there was a trend toward increased neuronal migration at day 14 and the majority of BrdU-labeled cells co-staining with NeuN at day 28. Conclusions: Intranasal delivery of TGF $\beta 1$ promotes neuronal migration and differentiation in SVZ of the adult mouse brain after focal cerebral ischemia. This may have therapeutic implications for cerebrovascular disease.

\section{Neuroprotective Effects of Edaravone against Hypoxia-Reoxygenation Injury in Cultured Hippocampal Cells}

\author{
W. Wang, T. Wu, X. Ding, H. Xiao, H. Zhang \\ Department of Neurology, the First Affiliated Hospital and \\ Key Laboratory of Applied Toxicology of Nanjing Medical \\ University, Nanjing, China
}

Background: The reactive oxygen species and $\mathrm{Ca}^{2+}$ overload play a critical role in ischemia/reperfusion ( $\mathrm{I} / \mathrm{R})$ injury. Edaravone has potent effects in the brain as a free radical scavenger in ischemia-reperfusion. In this study, we used cultured hippocampal neurons as a model to demonstrate the protective effects of edaravone against neuronal ischemia/reperfusion injury and elucidate the underlying mechanisms. Methods: Acute glucose-oxygen deprivation and subsequent reoxygenation were used to model ischemia/reperfusion injury in cultured hippocampal cells. Cells were treated with several concentrations of edaravone upon reoxygenation. Malondialdehyde (MDA) content and superoxide dismutase (SOD) activity were measured by assay kits. The intracellular $\left[\mathrm{Ca}^{+}\right]$and mitochondria membrane potential were determined by laser scanning confocal microscopy. The rate of apoptosis was detected by flow cytometry. Results: Edaravone reduced malondialdehyde level and raised the SOD activity when applied upon reoxygenation in a dose-dependent manner compared with untreated group. The peak neuroprotective effects occurred at 100 and $300 \mu \mathrm{mol} / 1$. $\left[\mathrm{Ca}^{2+}\right]_{\mathrm{i}}$ was significantly reduced in $100 \mu \mathrm{mol} / \mathrm{L}$ edaravone-treated group than in untreated group $(32.5 \pm 4.0$ vs. $50.2 \pm 3.6, \mathrm{p}<0.01)$. Treatment with $100 \mu \mathrm{mol} / \mathrm{L}$ edaravone significantly inhibited the decrease of mitochondria membrane potential after hypoxia/reoxygenation $(204 \pm 11.6 \%$ compared with untreated group, $\mathrm{p}<0.01$ ). Cell apoptotic rate was significantly decreased following edaravone treatment from $33.7 \pm 2.3 \%$ (untreated group) to $16.6 \pm 1.4 \%(100 \mu \mathrm{mol} / \mathrm{l}$ edaravone treated group). There was no significantly protective difference between 100 and $300 \mu \mathrm{mol} / 1$ edaravone. Conclusions: Edaravone effectively protects neuron injury after ischemia/reperfusion by inhibiting lipid peroxidation, reducing $\mathrm{Ca}^{2+}$ overload, elevating mitochondria membrane potential, and decreasing apoptosis.

\section{Qualitative Distribution of Cerebrolysin in the CNS following Intranasal Delivery}

\author{
X. Liu, G. Xu, W. Zhu, W. Wu \\ Department of Neurology, Jinling Hospital, Nanjing, China
}

Objective: Qualitatively determine the distribution of radiolabeled Cerebrolysin in the CNS after intranasal and intravenous delivery. Methods: Cerebrolysin was radiolabeled with ${ }^{125} \mathrm{I}$. An average of $0.98 \mathrm{mg}(46 \mu \mathrm{Ci})$ of a mixture of labeled and unlabeled Cerebrolysin and was either intranasally or intravenously delivered to each anesthetized and tracheotomized rat. The descending aorta was cannulated for blood sampling and perfusion-fixation. Intranasal delivery, Cerebrolysin was delivered over approximately $30 \mathrm{~min}$ as $10 \mu \mathrm{l}$ drops (total $148 \mu \mathrm{l}$ ) in alternating nares. For intravenous delivery, Cerebrolysin was delivered by a bolus injection into a cannula in the femoral artery in a total of $0.5 \mathrm{ml}$ saline $(0.9 \% \mathrm{NaCl})$ over $1 \mathrm{~min}$. Blood sample $(0.2 \mathrm{ml})$ was drawn every 5 min during delivery. After drug delivery, rats were perfused with $4 \%$ paraformaldehyde; the brain was removed and sliced into either 1-mm sagitall or coronal sections. A phosphor screen was placed over the tissue for 28 days and developed using and analyzed. Concentrations of Cerebrolysin in each blood sample and peripheral tissue sample were calculated using $\gamma$ counting. Results: Autoradiography in coronal section, intranasal administration resulted in excellent delivery of Cerebrolysin to most areas of the brain and spinal cord, and demonstrated much higher delivery of intranasal Cerebrolysin than intravenous Cerebrolysin to the central nervous system (CNS). Both coronal and sagittal sections show a decreasing gradient from the rostral to the caudal part of the brain following intranasal delivery. Direct comparison of two intranasal brains and one intravenous brain demonstrates significantly greater drug distribution in the intranasal coronal and sagittal section of brain and similar drug distribution between the two intranasal coronal and sagittal section of brains. $\gamma$ counting comparison of intravenous vs. intranasal administration of Cerebrolysin in 10 rats demonstrates greater delivery to the CNS with intranasal delivery $(p<0.01)$ and greater delivery to the peripheral organs and blood with intravenous delivery $(\mathrm{p}<0.01)$. On average, intravenous blood concentration were 6.8 times greater than intranasal blood concentrations. Five CNS tissues were collected and $\gamma$ counted including ventral brain dura, dorsal brain dura, optic nerve, trigeminal nerve, cervical spinal cord, olfactory epithelium, deep cervical lymph nodes and common carotid had significantly greater delivery following intranasal administration than intravenous delivery $(\mathrm{p}<0.01)$. All peripheral tissues (except superficial lymph nodes, esophagus, and trachea) had significantly greater concentration following intravenous delivery $(\mathrm{p}<0.01)$. Conclusions: Overall, these results demonstrate significantly greater delivery to the brain following intranasal administration of Cerebrolysin. Intranasal Cerebrolysin is directly delivered to the brain along the olfactory and trigeminal pathways. Intranasal administration 
of Cerebrolysin will provide a non-invasive method to treat Alzheimer's disease and stroke patients by providing larger drug concentrations to the brain and minimal concentrations to the peripheral organs.

\section{Intranasal Administration of Nerve Growth Factor Protects against Sarin-Induced Brain Damage}

\author{
X. Fan, W. Zhu, G. Xu, R. Zhang, X. Fan, X. Liu \\ Department of Neurology, Jinling Hospital, Nanjing \\ University School of Medicine, Nanjing, China
}

Background and purpose: Sarin, an irreversible acetylcholinesterase inhibitor, can induce severe and long-term brain damage following status epilepticus in rats, which is most prominent in piriform cortex, hippocampus and amygdala. Presently the conventional therapy (atropine, pralidoxime et al) does not completely avoid this damage. Nerve growth factor (NGF) has the neuroprotective effect on nerve injury induced by many etiological factors. But intravenous injection of NGF can not cross the blood-brain barrier (BBB) efficiently. And intracerebroventricular injection has risks of infection and other complications. It was confirmed that NGF could be delivered to the brain directly following intranasal (IN) administration, bypassing the BBB. This study was aimed to investigate the effect of IN NGF on the main damage regions of sarin-poisoned rats. Methods: Sprague-Dawley rats were treated with $2 \mathrm{LD}_{50}$ of sarin (intramuscular injection) and administered by peritoneal injection of atropine sulphate, pralidoxime $1 \mathrm{~min}$ after intoxication. NGF or saline was administered via the olfactory pathway. The rats were sacrificed $24 \mathrm{~h}$ after sarin exposure. Damaged and residual healthy neurons were estimated and quantified on brain sections of piriform cortex, hippocampus and amygdala using hematoxylin-eosin (H\&E) staining, TUNEL staining and neuronal nuclei antigen (NeuN) immunohistochemistry. Results: The degree of sarin-induced brain injury was better in rats administered by intranasal with NGF than rats with saline. As assessed by H\&E staining, a massive quantity of degenerating neurons was seen in the region of interest of rats with IN saline, nor of rats with IN NGF. Compared to IN saline, IN NGF significantly reduces the number of apoptosis cells stained by TUNEL staining and increases the number of NeuN-positive neurons $(p<0.05)$. Conclusions: Intranasal delivery of NGF could reach the central nerve system efficiently; improve neuropathology following sarin-exposure rats. NGF administered by intranasal, a noninvasive and cost-effective method, should be a potential treatment for sarin intoxication.

\section{Cerebral Artery Angioplasty and Stenting: A Single-Center Experience from China}

\author{
X. Liu, G. Xu, R. Zhang, Q. Yin \\ Department of Neurology, Jinling Hospital, Nanjing \\ University School of Medicine, Nanjing, China
}

Objective: We present the results of the first 134 patients who underwent carotid angioplasty and stenting at Jinling Hospital, Nanjing
University between January 2003 and January 2006. Method: Data were collected on 103 patients who received a cerebral artery angioplasty and stent due to symptomatic carotid stenosis of $\geq 70 \%$ of the lumen at our hospital. Outcome measures were complications within 30 days following intervention, and restenosis 6 months after the procedures. Results: Intravascular stents were deployed in the common carotid arteries (8) and extracranial internal carotid arteries (65) of both symptomatic (50 patients) and asymptomatic patients (15 patients) with $>60 \%$ stenosis ( 58 with $>70 \%$ and 7 with $60 \%$ stenosis). Nine patients underwent bilateral stent implantation. Distal brain protection devices were used in 56 patients. Angioplasty and stenting were performed in vertebral artery in 31 patients, and 4 patients underwent bilateral vertebral arteries stenting. There are 3 patients underwent a carotid stenting and a vertebral artery stenting. Two patients underwent basilar artery angioplasty and stenting. Thirty-one patients underwent intracranial carotid or middle cerebral artery angioplasty and stenting. Mean admission time was 7 days. Follow-up for 6 months employed clinical evaluation, duplex scanning at 3 and 6 months. CT or MRI scanning were performed before and 3 months after the procedure. Angiographic success, defined as maximal residual stenosis $<30 \%$, was achieved in $100 \%$. Clinical success, defined as maximal residual stenosis $<30 \%$ and absence of death or recurrent transient ischaemic attack or stroke in hospital, was achieved in $96.2 \%$ of treated cases. Complications included one procedurerelated ipsilateral miner stroke, one locked-in syndrome in a basilar artery stenting patient, and a transient ischemic attack in carotid stenting patient. No deaths occurred during the hospitalization. No early or late restenosis have been observed to date. Conclusion: This experience indicates that interventional neurovascular therapies may provide a useful alternative for selected patients requiring endoluminal reconstruction of cerebral artery stenosis.

\section{Risks Factors for Symptomatic Vasospasm after Subarachnoid Hemorrhage}

\author{
X. Yang, F. Cao, W. Liu, X. Zheng, H. Shen, W. Fu \\ Department of Neurosurgy, Second Affiliated Hospital, \\ Zhe Jiang University, Hang Zhou, China
}

Objective: To evaluated the independent risk factors of the occurrence of symptomatic vasospasm following aneurysmal subarachnoid hemorrhage. Methods: Symptomatic vasospasm was defined as the association of deterioration in a patient's neurological condition between 3 and 14 days after SAH with no other explanation. The clinical data of 186 cases with aneurysmal subarachnoid hemorrhage, including demographic, clinical and neuroimaging characteristics were reviewed retrospectively. The risk factors were analysed statistically. Results: Out of 186 cases, 29.6\% (55) developed symptomatic vasospasm. There was significant higher incidence of symptomatic vasospasm in patients with Fisher Grade III, poor neurological status at admission and repeated reoccurrence SAH $(\mathrm{p}<0.01)$. The age, sex, treatment modality and use of antifibrinolytic drugs (AFD) did not influence the development of symptomatic vasospasm $(\mathrm{p}>0.05)$. Multivariate analysis showed there were three independent risk factors: thickness of subarachnoid clot on CT scan (Odds Ratio [OR] 2.549, 95\% confidence interval [CI] 1.406 4.517), poor neurological status at admission (OR 2.342, 95\%, CI 1.320 4.159) 
and repeated reoccurrence SAH (OR 2.492, 95\%, CI 1.394 4.448). Conclusion: Fisher Grade III, poor neurological status at admission and repeated reoccurrence of SAH are significant independent risk factors of symptomatic vasospasm.

\section{Direct Transport of Transforming Growth Factor $\beta 1$ from the Nasal Cavity to Brain}

Y. Ma, X. Liu, G. Xu, H. Zhang

Department of Neurology, Jinling Hospital, Nanjing

University School of Medicine, Nanjing, China

Introduction: Transforming growth factor $\beta 1$ (TGF- $\beta 1$ ) plays an important role in the central nervous system. Recent studies revealed that exogenous administered TGF- $\beta 1$ protected against ischemic brain injury. However, circulating TGF- $\beta 1$ could not cross the intact blood-brain barrier (BBB), and intracerebroventricular administration is not clinical practical, thus its therapeutic potential is limited. In this study, we evaluated whether TGF- $\beta 1$ can be delivered into the brain following intranasal administration. Methods: Adult Sprague-Dawley rats were randomized into three groups, given recombinant human TGF- $\beta 1$ (rhTGF- $\beta 1$ ) intranasally or intravenously or given vehicle intranasally. Thirty minutes after administration, rats were killed following cerebrospinal fluid (CSF) and blood sample collections, then the brains were removed. The rhTGF- $\beta 1$ concentrations were analyzed by enzyme-linked immunosorbent assay (ELISA). Results: Following intranasal administration, rhTGF- $\beta 1$ was nonuniformly distributed in the brain, with concentrations in the olfactory bulb significantly higher than other regions of the brain, while limited rhTGF- $\beta 1$ was found in the CSF and blood samples. Little or no rhTGF- $\beta 1$ was detected in the brain following intravenous administration. Conclusions: While rhTGF- $\beta 1$ can not penetrate the $\mathrm{BBB}$, it reaches the brain rapidly from the nasal cavity bypassing the BBB, which may be associated with the extracellular transport through clefts between sustentacular cells and olfactory neurons. Therefore, the nasal pathway is a convenient, non-invasive route for direct drug delivery to the brain. Intranasal administration of TGF- $\beta 1$ may provide an effective way for the treatments of ischemic stroke and other central nervous system diseases.

\section{A Short-Term Assessment of Recombinant Human Granulocyte Colony-Stimulating Factor (rhG-CSF) in the Treatment of Acute Cerebral Infarction}

\author{
J. Zhang, M. Deng, Y. Zhang, W. Sui, L. Wang, A. Sun, \\ H. Song, M. Lu, D. Fan \\ Department of Neurology, Peking University Third \\ Hospital, Beijing, China
}

Background: In humans, bone marrow stem cells can be mobilized into the circulation using recombinant human granulocyte colony-stimulating factor (rhG-CSF), an approach that is effective in experimental stroke. And randomized placebo-controlled pilot trials
(STEMS) are performing by Sprigg et al. Objective: To assess the safety and efficacy of rhG-CSF in treatment of acute cerebral infarction by a randomized and placebo-controlled clinical trial. Method: 45 cases with acute cerebral infarction were randomly allocated to receive treatment by either rhG-CSF or placebo within 1 week of stroke onset. Among them, 15 cases were consecutively given rhGCSF $2 \mathrm{~g} / \mathrm{kg}$ for 5 days by subcutaneous injection. The other 30 cases were treated with placebo. The end points included NIH Stroke Scale and adverse reaction. Results: On the 5th day in rhG-CSF treatment, there was markedly increase of WBC count, and CD34 + cell proportion $(>1 \%$, i.e. $>106)$. For the treatment group, there was no significant difference between NIHSS score on the 10th day and that before treatment, but there was on the 20th day $(p=0.004)$. For parallel group, no significant differences in NIHSS scores on both the 10 th and 20th day compared with those before treatment. There were no significant differences between the two groups in terms of their respective NIHSS scores on the 10th and 20th day. No definite side effects were found in the treatment group during the 20 days. Conclusion: There was a statistical difference on the 20th day during the treatment of acute cerebral infarction with rhG-CSF, showing that rhG-CSF is an effective drug in the treatment of acute cerebral infarction. Finding no statistical difference on the 10th day means that the efficacy of rhG-CSF can only be seen in the long run. Although there was no statistical difference between the two groups, the gap has widening tendency with time until the 20th day.

\section{Survey of Risk Factors of Stroke and Evaluation of Stroke Risks in a Rural Community Population}

\author{
J. Guo, J. Huang, Y. Yang, G. Wang
}

Shanghai Institute of Cerebrovascular Disease Prevention and Cure, Shanghai, China

Background: Exposure of risk factors of stroke result in functional and structural disorder of cerebrovascular. Hemodynamic is an indicator of the disorder. Analysis of risk factors of stroke and cerebrovascular hemodynamic indexes (CVHI) make for evaluation relative risk of stroke (RR). Objective: To analyze and evaluate risk factors of stroke in rural community population and describe population distribution. Methods: Cross-sectional design. Participants aged $\geq 40$ year and free of stroke were selected by cluster sampling in a rural community. There were 5,335 (male 2,234, female 3,101) individuals enlisted in the study and its means of age was $56.3 \pm 11.51$ (40 96) year. Survey of risk factors of stroke and examination of CVHI were curry out. CVHI was detected using CBA CV-300 CVHI instrument. Methods of risk factors investigation, CVHI score synthesizing and estimation of RR were the same as reference. Results: Exposure rate of hypertension, heart disease, diabetes, obese, family history of stroke, cigarette smoke and alcohol drink were $31.74 \%$, $6.09 \%, 1.16 \%, 3.63 \%, 3.22 \%, 86.66 \%$ and $40.87 \%$ in male and $32.76 \%, 9.22 \%, 1.55 \%, 4.35 \%, 3.84 \%, 0.19 \%$ and $0.13 \%$ in female. There were significant differences between male and female of hypertension, heart disease, smoking and drink $(p<0.01)$. Exposure rate of hypertension and heart disease, and level of systolic blood pressure were increased with aging. However, BMI and CVHI were decreased with aging. The rate of RR of stroke increased to 5,7 and 14 was 
going up with aging, and it was $9.13 \%, 5.68 \%, 3.98 \%$ in male and $12.87 \%, 6.26 \%, 3.99 \%$ in female $(p<0.01)$. Conclusions: Risk factor exposure of stroke in Shanghai rural community population is with age and gender distributional differences and hypertension is the major factor. There are about $20 \%$ individuals in existence injury of cerebrovascular function and significant increase of stroke risks.

\section{Risk Factors and Predilection Sites of Carotid Artery Atherosclerotic Stenosis in an Urban High-Risk Population in Wuhan, China}

G. Chen, X. Qiu, X. Wang, B. Liu

Department of Neurology, Integrated Traditional Chinese and Western Medicine Hospital of Tongji, Medical College of Huazhong University of Science and Technology,

Wuhan, China

Objectives: To explore the association of Carotid artery atherosclerotic stenosis and risk factors, predilection sites of carotid atherosclerosis in an urban high risk population in Wuhan, China. Methods: 105 high risk patients aged 35-84 were examined. Intracranial and extracranial Artery atherosclerotic stenosis was measured using DSA. The association of carotid artery atherosclerotic stenosis and risk of carotid atherosclerosis was analyzed using Logistic regression analyses. Results: Incidence of high blood pressure, high serum cholesterol and diabetes of carotid atherosclerosis group were higher than control group. Carotid atherosclerosis was significantly correlated with smoking, sex, high blood pressure, high serum cholesterol, diabetes and family history of stroke. The OR of high blood pressure, high serum cholesterol and diabetes is 10.595 , the OR of smoking is 2.765 , the OR of sex is 0.883 , and the OR of heredity is 0.843 . Among predilection sites of Carotid artery atherosclerotic stenosis, stenosis of common origin of carotid artery was $35 \%$, stenosis of origin of internal carotid artery was $40 \%$. Conclusions: A history of high blood pressure, high serum cholesterol and diabetes was the most dangerous factor to carotid artery atherosclerotic stenosis. Smoking was the second risk factor. Furthermore, Family history of stroke was a risk factor similar to sex factor. Common origin of carotid artery and origin of internal carotid artery were the most frequent predilection sites.

\section{Intranasal Administrating Basic Fibroblast Growth Factor to Patients with Acute Ischemic Stroke}

\author{
G. Xu, X. Liu, W. Wu, X. Fan \\ Department of Neurology, Jinling Hosptial, Nanjing \\ University School of Medicine, Nanjing, China
}

Background and objective: Basic fibroblast growth factor (bFGF) is up-regulated in the brain and conduces to neuroprotection and angiogenesis in experimental brain ischemia. In this study, we evaluated the efficacy and safety of intranasal administrated bFGF in patients with acute cerebral infarction. Methods: Sixty-four patients with acute cerebral infarction, who had been evaluated by a neurologist within $6 \mathrm{~h}$ after the onset of stroke, were enrolled as subjects. Patients were randomized into two groups, control group (32 patients) and bFGF treated group (32 patients). Patients in bFGF group received intranasal administration of $1000 \mathrm{IU}$ bFGF (dissolved in $1 \mathrm{ml}$ normal saline) once daily for 10 days. Patients in control group receive intranasal $1 \mathrm{ml}$ normal saline as placebo. There were no significant differences concerning the patients age, stroke severity and time course, major risk factors for stroke in the two treatment groups $(p>0.05)$. Beside bFGF and its placebo, all patients were treated following the guidelines for management of ischemic stroke. All patients received MRI or CT scans before and after the treatment, and were followed for 3 months. Neurological and cognitive functions were evaluated serially in the two treatment groups. Results: Three months after the onset of stroke, patients in bFGF group had significantly better neurological functions compared with control group $(p<0.05)$. The index of neurological function defection in the control group and the bFGF group were $21.9 \pm 8.31$ and $22.7 \pm 7.4$ respectively before treatment $(\mathrm{p}>0.05)$, and it was decreased to $14.6 \pm 8.1$ and $10.3 \pm 7.9$ respectively after the treatment $(\mathrm{p}<0.05)$. The cognitive functions as evaluated by Mini-mental State Examination were also improved more significantly contrasted to placebo treated patients $(\mathrm{p}<0.05)$. Conclusion: Intranasal administrated bFGF can improve the recovery of cerebral infarction and reverse the cognitive impairment after cerebral ischemia.

\section{Microsurgical Anatomic Study of Vertebral Artery and the Blood Supply for Ventral Lateral of Medulla Oblongata}

\author{
J. Xiong, X. Zhang, R. Ji, X.-P. Zhou \\ Department of Neurosurgery, Xijing Hospital, Fourth \\ Military Medical University, Xi'an, China
}

Background: The surgical management of vertebral arterybasilar artery aneurysms remains a significant treatment challenge. Understanding the anatomical relationship between arteries and medulla oblongata is very important for a surgeon. The aim of the study is to provide morphological basis for the management of the vertebral artery-basilar artery aneurysms, which can protect the vessels in ventral lateral region of medulla oblongata. Anatomy of vertebral artery and the blood supply for ventral lateral of medulla oblongata was investigated in cadaveric heads. Materials and methods: Twenty four formalin-fixed adult Chinese cadaveric heads were dissected with the aid of microscope. The course of the vertebral artery from the $\mathrm{C} 2$ transverse foramen to its dural entry was studied. The distance of vertebral artery from lateral side of transverse foramen of atlas to its dural entry was measured. Then the intracranial section of the vertebral artery was investigated after remove of the dura. The small nutrient arteries entry the medulla oblongata was examined. The source, situation, length and outer diameter of nutrient arteries of ventral lateral region of medulla oblongata were investigated. Result: The distance of vertebral artery from lateral side of transverse foramen of atlas to its dural entry was $16.87 \pm 2.08$ (12.64-20.1) mm (Left), $16.79 \pm 1.90$ (12.44-20.22) mm (Right). The external diameters of the vertebral artery at the level of the transverse 
foramen of atlas were $5.10 \pm 1.01(3.06-7.24) \mathrm{mm}$ (Left), $4.89 \pm 1.73(3.04-10.40) \mathrm{mm}$ (Right). There was rich nutrient artery entry the medulla oblongata. In 48 sides dissected specimen, there were nutrient arteries with diameter above $0.2 \mathrm{~mm} 162$ branches: the numbers which come from anterior inferior cerebellar artery, vertebral artery, basilar artery and posterior inferior cerebellar artery were $48(29.6 \%), 45(27.8 \%), 39(24.1 \%)$ and $30(18.5 \%)$, respectively. Conclusion: The microanatomy of vertebral artery provides important parameters for neurosurgeons. Understanding the blood supply of ventral lateral region of medulla oblongata can also help surgeon to protect these nutrient arteries in the VA-BA aneurysm operation.

\section{The Diagnostic and Predictive Value of QEEG in Patients with Acute Supratentorial Cerebral Infarction}

\author{
W. Dong, Y. Liu \\ Department of Neurology, First Affiliated Hospital of \\ Soocow University, Suzhou, China
}

Objectives: To monitor patients with acute supratentorial cerebral infarction (ASCI) within $48 \mathrm{~h}$ of onset with EEG and observe their change of EmEEG and qEEG. This study was designed to investigate their diagnostic and predictive value. Methods: Forty-two inpatients with acute cerebral infarction were collected. Chose the first $30 \mathrm{~min}$ artifact-free segment of CEEG as emergency-EEG (EmEEG), and compare its diagnostic value with CT. For 25 cases with ASCI, by means of fast Fourier transform (FFT) mean values of relative power were calculated from the first artifact-free EEG for $120 \mathrm{~s}$ of each EmEEG. $\Delta$ (2-3.8 Hz), $\theta(4-7.8 \mathrm{~Hz}), \alpha-1(8-8.9 \mathrm{~Hz}), \alpha-$ $2(9-10.9 \mathrm{~Hz}), \alpha-3(11-12.8 \mathrm{~Hz})$ or $\beta(13-30 \mathrm{~Hz})$ bands was gotten for each channel and then its preponderant frequency was found out. The diagnostic value of EmEEG and BEAM were matched. To 14 patients within $24 \mathrm{~h}$ of onset, the relative power changes of $\Delta$ waveband were analyzed one hour apart for the first 5-7 h after CEEG, and the acute $\Delta$ change index (aDCI) was worked out. Results: EmEEG revealed abnormalities in 30 of 42 cases $(79.4 \%)$, while the first CT in 25 of 42 cases. For cases with supratentorial infarct the EmEEG was more valuable in showing focal alterations than those with lacunar infarct. Along with the time elapsing, the positive rate of CT increased, while EmEEG's advantage weakened. For patients with supratentorial cerebral infarction, the positive rate of BEAM was $92.0 \%$, CT $72.0 \%$ and EmEEG $76.0 \%$. The preponderant frequency is downtrend, with the increase in $\Delta$ waveband and decreases in $\alpha-1$ and $\alpha-2$ wavebands at the lesion hemisphere. The aDCI was significantly correlated with the 28th day's NIHSSS $(\mathrm{p}=0.92, \mathrm{p}<0.01)$. Conclusions: BEAM was more efficient and superior in revealing focal abnormalities in the patients with ASCI appeared normal by CT. The change of relative power in $\Delta$ band is more useful in localizing ischemic side and even the focus. Negative aDCI indicates good outcome while positive indicates bad one.

\section{The Study on Bone Marrow Stromal Cells Transfected with Noggin Gene Differentiating into Neuron-Like Cells in vitro}

\author{
S. Zhou, X. Wei, M. Zhang, L. Liu, Y. Wang \\ Department of Neurology, Qilu Hospital of Shandong \\ University, Jinan, China
}

Objective: To construct an expression vector of Noggin and explore the differetiation of BMSC transfected with this vector. Methods: (1) From the brain tissue of a normal human fetal, noggin gene was amplified by reverse transcription-polymerase chain reaction (RT-PCR). By employing gene engineering techniques, the gene was recombinated to the new vector pCS2 $+[\mathrm{T} \alpha 1]-$ Noggin. (2) The rats' bone marrow stromal stem cells were isolated by density gradient contrifugation with Percoll. The fourth and sixth generation of BMSCs was transfected the expression vector with Noggin by lipofectin. After culturation, the differentiated cells was detected the expresson of NSE, MAP-2 but no GFAP by immunocytochemistry. Results: (1) Analysis of the sequence results showed that the full length of the fragment cloned from brain tissue of human fetal was correct and the recombinant $\mathrm{pCS} 2+[\mathrm{T} \alpha 1]-$ Noggin plasmid was successfully constructed. (2) The BMSC transfected the vector displayed the neuron morphologies with long and multipolar projections. It was confirmed that the differetiated cells expressed NSE, MAP-2 but no GFAP by immunocytochemistry. Conclusions: The bone marrow stromal cells transfected with Noggin gene differen-tiate into neuronlike cells in vitro.

\section{The Retrospective Clinical and MR Analysis of 58 Patients with Posterior Circulation Ischemia}

W. Zhao, X. He

Department of neurology, 81st hospital, Nanjing, Jiang Su, China

Objective: To investigate the probable etiology and preventive measurement to control posterior circulation ischemia (PCI). Methods: Fifty eight patients with posterior circulation ischemia were analyzed retrospectively, including clinical and radiological data. Results: Verterobasilar artery (VBA) imaging showed $82 \%$ patients with PCI had an asymmetry Vertebral Arteries (VA), of which $24 \%$ had different degree stenosis in the branch of VA, $3 \%$ had an occlusion of VA. Fifty two infarctions were observed in brain stem, cerebellum, and occipital lobar respectively. Of them, 38\% were fresh, $72 \%$ were dated. Among the fresh infarctions, $75 \%$ were responsible. $70 \%$ patients with PCI were found with cervical bone degeneration of different degrees. Only $3 \%$ were found with a V2 cervical circuitry of VA. Conclusion: It is basis to lay more emphasis on preventing and controlling the vascular atherosclerosis on thinner blood vessels of BVA, yet as for patients with stenosis which led to obvious ischemia, intravascular therapy will play an increasing role in controlling exacerbations of symptoms resulted from PCI. 


\section{Research on Vascular Remodeling and Changes of Cerebrovascular Hemodynamic Index in Rat Carotid}

\author{
X. Zhang, W. Oiu, G. Wang \\ Shanghai Cerebrovascular Disease Prevention and \\ Treatment Research Institute, Shanghai, China
}

Objective: To investigate changes of histomorphology and cerebrovascular homodynamic indexes (CVHI) during vascular remodeling in different-aged rats. Methods: Morphometry and microstructure of carotid artery in 3-month-old, 12-month-old and 24-month-old Sprague-Dawley rats were studied quantitatively by histological method and computer image analysis; expressions of collagen I, II and elastin were studied by immunohistochemistry staining technique in vascular wall tissues and changes of CVHI were observed by cerebrovascular homodynamic analyzer in rats. Results: Our experimental results showed that arterial geometric shape was remodeling and arterial compliance was lower in rat carotid, and expressions of collagen I, II and elastin decreased with age increasing. Compared with 6-month-old and 12-month-old rats, the carotid artery maximal velocity (Vmax), minimal velocity (Vmin) were significantly decreased $(\mathrm{p}<0.05)$, but the cerebrovascular peripheral resistance $(\mathrm{Rv})$, characteristic impedance $(\mathrm{Zcv})$ and pulse wave velocity $(\mathrm{Wv})$ were significantly increased $(\mathrm{p}<0.05)$ in the 24 -month-old rats. Conclusions: It was significant differences in vascular histomorphology and component and CVHI during carotid remodeling in rats. CVHI were sensitive index to evaluate vascular state during vascular remodeling.

\section{Deliverying BDNF Gene into CNS by Recombinant Herpes-Simplex-Virus Vectors via Intranasal Olfactory Pathway in Rats with Focal Cerebral Ischemia}

\author{
G. Xu, H. Zhang, W. Zhu, M. Ma
}

Department of Neurology, Jinling Hosptial, Nanjing University School of Medicine, Nanjing, Jiangsu Province, China

Objective: Several growth factors such as brain-derived neurotrophic factor (BDNF) have been reported to be effective for neurogenesis and neuroprotecton after focal cerebral ischemia in animal model. But gene therapy for cerebrovascular disease in human still has many problems, of which lacking an effective delivery pathway to central nervous system (CNS) is a major hindrance for the clinical application of this promising strategy. This study was aimed to evaluate the feasibilty and efficacy of deliverying BDNF gene with recombinant herpes-simplex-virus vectors to CNS via intranasal olfactory pathway in rat with focal cerebral ischemia. Method: Recombinant herpes-simplex-virus vector was constracted by deleting 6 viral genes, which included the coding sequence for an immediat early (IE) gene, ICP4. Focal cerebral ischemia was induced by middle cerebral artery occlusion (MCAO) with a nylon filament in Sprague-Dawley rats. MCAO rats were randomized into three groups: BDNF, eGFP and control. Six hours after MCAO, recombinant herpes-simplex- virus vectors carrying BDNF gene or enhanced green fluorescent protein (eGFP) gene, a marker gene, or the void vectors were administrated intranasally. Bromodeoxyuridine (BrdU) was injected intraperitoneally twice daily on the fifth and sixth days for the purpose of neurogenesis measurement. On the seventh day and forteenth day after MCAO, the cerebral infarct volumes, neurogenesis, expression of BDNF and eGFP were assessed. Neurological outcomes were serially assessed by the rotated test after MCAO. Results: Rats in BDNF group scored higher in neurological function tests 7 and 14 days after MCAO compared with eGFP and control groups $(p<0.05)$. Rats administrated with BDNF gene has a trend of decreased infarct volume 14 days after MCAO compared with that of eGFP and control groups, but the differences did not reach the significant level $(p=0.065)$. Histological detections revealed that expression of BDNF in hippocampus and temporal lobe were significantly enhenced 7 and 14 days after MCAO in BDNF group in contrast to eGFP and control groups $(p<0.05)$. Green fluorescence was also detected in hippocampus and temporal lobe of rats administrated with eGFP gene. The BrdU-positive cells in subventricular zone and hippocampus was significantly increased in BDNF gene treated rats than eGFP treated and control rats $(\mathrm{p}<0.05)$. Conclusions: Recombinant herpes-simplex-virus vectors administrated via intranasal olfactory pathway can efficiently delivering BDNF gene into CNS and expressing. BDNF gene therapy is efficacious in treating focal cerebral ischemia in rats.

\section{MR-PWI Perfusion and Cerebrovascular Reservation in TIA Patients with Intracranial Stenosis}

\author{
L. Huang, X. Song, L. Huang, Y. Wang, R. Hu, H. Zhou \\ Neurology Department, First Affiliated Hospital, Jinan \\ University, Guangzhou, Guangdong, China
}

Objective: To study the brain perfusion and vascular reserve in patients who had TIA with stenosis of the intracranial arteries. Method: Four TIA patients who had been confirmed stenosis of the intracranial arteries by MRA and/or DSA, were examined basic PWI. Two days later they took Diamox $2 \mathrm{~g}$ orally, and then followed by another PWI examination $2 \mathrm{~h}$ later. We compare the parameter in PWI before and after Diamox. Result: Two patients showed decreased brain perfusion in basic PWI, after Diamox they showed more decreased brain perfusion. One patient had no decreased brain perfusion before and after Diamox. One patient showed decreased brain perfusion in right fronto-parietal region, and after Diamox it showed normal perfusion almost the same on both sides. Conclusion: Using Diamox before and after PWI can show brain perfusion and vascular reserve, and it will provide the reliance for diagnosis and treatment of stenosis of the intracranial arteries in the TIA patients. 


\section{Early Intervention Study of Xingnaojing Injection on Acute Severe Cerebral Hemorrhage Leading to Systemic Inflammatory Response Syndrome}

\author{
L. Wang, H. Guo, S. Xiang, L. Ji \\ Subsidiary Second Hospital of the Chinese Medicine \\ University of Shandong, Shandong Jinan, China
}

Objective: To study the early intervention therapeutic efficacy of Xingnaojing injection on acute severe cerebral hemorrhage leading to systemic inflammatory response syndrome, observe the changes of serum TNF- $\alpha$, IL-1 before and after treatment of Xingnaojing injection. Methods: Sixty cases were randomized into two groups: Xingnaojing injection group (30 cases), aceglutamide group (30 cases). By using ELISA method, the serum TNF- $\alpha$, IL- 1 level was measured before treatment (within $48 \mathrm{~h}$ of acute severe cerebral hemorrhage) after 3 days/ 7 days/14 days. Results: (1) The serum TNF- $\alpha$, IL-1 level was significantly higher in the two observed groups than normal control before treatment $(\mathrm{p}<0.01)$. (2) In Xingnaojing injection group, the serum TNF- $\alpha$, IL-1 level was significantly decreased after treatment at 3 days $(p<0.05)$, and was decreased most obviously after treatment at 7 days $(\mathrm{p}<0.01)$. The serum TNF- $\alpha$, IL-1 level was normal after treatment at 14 days. In aceglutamide group, the serum TNF- $\alpha$, IL-1 level was upgraded after treatment at 3 days $(\mathrm{p}<0.01)$, and was decreased after treatment at 7 days. The serum TNF- $\alpha$, IL-1 level was significantly increased after treatment at 14 days than promedication. (3) The incidence of MODS in Xingnaojing injection group was $2 / 30$, and that in aceglutamide group was $11 / 30$. Conclusion: Xingnaojing injection can significantly inhibit the release of cytokine after acute severe cerebral hemorrhage and decrease the incidence of MODS.

\section{MR Diffusion Tensor Imaging: Correlation Study of the Fiber Tracts Integrity Perihematomas and Functional Recovery after ICH}

W. Wang, X. Zhang, X. Yin, C. Li

Departments of Radiology and Neurology, Yangzhou No.1 People's Hospital, Yangzhou University, Yangzhou, China

Background and purpose: Recently developed diffusion tensor imaging (DTI) and diffusion tensor tractography (DTT) techniques provide new methods to observe neural fiber tracts in vivo. The purpose of this study is to investigate acute ICH (intracerebral hemorrhage) affection to perihematomal neural fiber tracts by using diffusion tensor imaging (DTI), and to evaluate its value to the clinical prognosis. Methods: DTI was performed on 25 patients who were within 2 weeks after the upsets of basal ganglia or thalamus hemorrhage. The integrity of the fiber tracts, including pyramidal tracts and arcuate fibers crossed the external capsule and optic radiations, was assessed by DTI and the DTT maps which were reconstructed by diffusion tensor tractography techniques. Follow-ups were carried out after 3 months, including motor function assessment with Barthel Index, optical space deletion and language evaluation in according to Stroke Impairment Assessment Set (SIAS). Results: Except 4 cases data of dissatisfaction, the integrity showed from DTI of the pyramidal tracts after acute ICH correlated well with BI scores after 3 months from upsets $(r=0.814, p=0.0001)$, the integrity of the left side arcuate fibers correlated SIAS scores for language $(\mathrm{r}=0.327, \mathrm{p}=0.043<0.05)$ and there was no correlation between the integrity of the optic radiations and SIAS scores for the optical space deletion $(\mathrm{p}>0.05)$. Conclusion: The implication status of the pyramidal tracts around the hematomas after acute ICH showed by DTI and DTT techniques can predict the recovery of motor function preferably. DTI provides a valuable method to assess the secondary injury and functional recovery of neural fiber tracts after ICH.

\section{Functional Outcomes After Dysphagia Therapy with Neuromuscular Electrical Stimulation}

\author{
M. OuYoung \\ Department of Speech Pathology, USC University \\ Hospital, Los Angeles, Calif., USA
}

Objective: To evaluate the safety and efficiency of utilizing conventional dysphagia treatment in conjunction with a neuromuscular electrical stimulation device (VitalStim) in treating a variety of patients during a two year period (2003-2005) at a university hospital outpatient setting. Methods: Thirty-three patients were evaluated via modified barium swallow study and received dysphasia therapy with VitalStim during December 2003-December 2005. VitalStim is currently the only FDA approved electrical stimulation program for swallowing. The age range of the patients was from 32 to 103 years of age. The etiology of the dysphasia was acute neurological (CVA) 15/33, progressive neurological (Parkinson's) 6/33, and head and neck cancer $12 / 33$. All patients received treatment from a licensed speech pathologist with a minimum of 5 years clinical experience in dysphasia evaluation and treatment. Before the start of treatment, patients received an objective swallow evaluation via a modified barium swallow study in the Radiology Department at USC University Hospital. The average number of treatment sessions was 15-20 sessions. Swallow levels were assigned using the ASHA Functional Communication Measure for Swallowing as well as the VitalStim scale for pre and post treatment levels of functioning Results: Findings indicated that $32 / 33$ patients who received dysphasia therapy in conjunction with a neuromuscular electrical stimulation demonstrated improvement by at least one functional level. One patient who did not demonstrate significant improvement was identified as having cricopharyngeal dysfunction and had not yet received a dilatation. Conclusion: This preliminary data suggests that dysphagia therapy with a neuromuscular electrical stimulation is a safe and effective therapy with patients who have dysphasia caused by a variety of diagnoses. 


\section{Difference between Anterior and Posterior Circulation Stroke Risk Factors}

\author{
S.A. Mousavi, T. Hoseini \\ Department of Neurology, Alzahara Hospital, Isfahan \\ University of Medical Sciences, Sofeh St., Isfahan, Iran
}

Background: Ischemic strokes occurring in the anterior circulation are the most common of all ischemic strokes. Strokes occurring in posterior circulation are more severe and grave clinicians have tended to view anterior circulation $(\mathrm{AC})$ and posterior circulation (PC) strokes as separate entities, with different underlying pathogenesis, natural histories, potential responsiveness to interventions such as anticoagulation and risk factors. We sought to explore differences between AC and PC stroke with regards to their risk factors. Methods: In this prospective descriptive study, we evaluated 250 patients that 125 had anterior circulation involvement and 125 had posterior circulation involvement referred by stroke to Alzahra and Noor University Hospitals and Private Clinic between January 2000 and December 2004. Stroke in Anterior Circulation and Posterior Circulation diagnosed by Clinical and Neuroimaging Findings include Brain CT Scanning and MRI. Data on pre stroke cerebrovascular risk factors for each patient were recorded from the records of the previous and present evaluation that included hypertension, diabetes mellitus, hyperlipidemia and smoking. Results: The average age of patients with Anterior circulation Stroke was $73 \pm 19.15$, and average age of patients with Posterior Circulation Stroke was $70 \pm 19.7$. Of 125 patients with anterior circulation stroke, 57 $(45.6 \%)$ of them were male and $68(54.4 \%)$ of them were female. Also of 125 patients with posterior circulation stroke: $54(43.2 \%)$ of them were male and $71(56.8 \%)$ of them were male. The prevalence of Hypertension as a major risk factor of stroke was higher in patients with Posterior Circulation Stroke in comparison to patients with Anterior Circulation Stroke (60\% vs. 40.8\%) and the OR for Posterior Circulation Stroke was 2.8 (95\% CI; 1.27-3.73). The prevalence of smoking in posterior circulation was higher than the anterior circulation Stroke (32.8\% vs. $15.2 \%)$ and this difference was statistically significant $(p=0.001)$. Discussion: In this study, the prevalence of hypertension as a major risk factor of stroke was higher in patients with posterior circulation stroke, but with regards to prevalence of diabetes mellitus as a risk factor for Stroke, there is not any difference between anterior circulation and posterior circulation stroke. Our results showed, hyperlipidemia was a risk factor for anterior and posterior circulation stroke and the prevalence of them was equal for both type of strokes, while the prevalence of smoking in posterior circulation was higher than the anterior circulation stroke.

\section{Effect of Intranasal Administrated Cerebrolysin on Ischemic Stroke}

\author{
X. Liu, G. Xu, W. Zhu, X. Fan \\ Department of Neurology, Jinling Hospital, Nanjing \\ University School of Medicine, Nanjing, China
}

Background and purpose: Cerebrolysin is a brain-derived peptide drug that increases the BBB-GLUT1 and MAP2 genes expression, thus exerting a neuroprotective effect. The present study was aimed at investigating in patients with ischemic stroke influence of Cerebrolysin infusions on cognitive performance, clinical outcome and electrophysiological results. Subjects and methods: Thirty patients were randomized into two groups. All patients receive standard management according to the established ischemic treatment guidelines. In addition, 15 patients receive intranasal administrated Cerebrolysin in dose of $1 \mathrm{ml}$ for 7 days after shortly after the onset of the ischemic stroke. The other 15 patients received same dosage of normal saline as placebo. Results: A decrease in slow electroencephalogram activity and an increase in fast frequencies were observed after the administration of Cerebrolysin. This electrophysiological effect was not influenced by stroke time course or severity, or by the chronic treatment with other neuroprotection agents. Cognitive performance, evaluated with Mini-mental state examination (MMSE), improved in ischemic stroke patients after Cerebrolysin treatment, independent of disease severity, time course or disability. A significant improvement in the patients' clinical outcome, only evident during the first year after the onset of stroke was also detected following Cerebrolysin infusions. No relevant changes in biological parameters nor drug-related adverse events were observed. Conclusion: These promising preliminary results suggest that intranasal Cerebrolysin might be a useful treatment to improve the recovery of patients with ischemic stroke, and encourage the conduction of confirmatory clinical trials.

\section{Drug-Eluting Stent Implantation for the Percutaneous Treatment of Stenosis in Intracranial Arteries and Extracranial Vertebral Arteries}

\section{Q. Huang, J. Liu, B. Hong, Y. Xu, R. Zhao, J. Chen}

Department of Neurosurgery, Changhai Hospital, Second Military Medical University, Shanghai, China

Objective: The aim of the present study was to evaluate the early results and mid-term outcomes following angioplasty of intracranial stenosis and extracranial vertebral stenosis with drugeluting stents. Methods: All of the patients presented with recurrent TIA or cerebral infarction with $>50 \%$ stenoses in intracranial arteries and extracranial vertebral arteries, and had failed maximal medical therapy. All of the lesions were treated with primary stenting under local or general anesthesia. Clopidogrel and aspirin were continued and patients had clinical follow-up and vascular imaging. Results: Of 53 patients (55 stenoses) with lesions in intracranial arteries (47 lesions) or extracranial vertebral arteries (8 lesions), 51 patitents (53 lesions) were successfully treated with 51 Cypher stents and 2 Taxus stents. The mean stenosis severity was reduced from $74.5 \pm 6.7 \%$ to $8.7 \pm 4.4 \%$. Three patients had inchemic events related to perforating arteries. During 2-14 months follow-up, the patients were clinically asymptomatic and had no recurrent TIA/stroke. Angiographic follow-up was obtained in 30 patients $(56.6 \%)$ at a mean of 6.4 months. No restenosis was observed in one patient $(3.3 \%)$. Conclusion: The results confirm the safety and effectiveness of using the DES in cerebrovascular diseases with a significantly lower risk of restenosis compared with the bare stent. 


\section{Perihematoma Damage at Different Time Points in Experimental Intracerebral Hemorrhage}

\author{
S. Zhang, X. Yin, X. Zhang, W. Wang \\ Department of Neurology, and Department of Radiology, \\ Tongji Hospital, Tongji Medical College, Huazhong \\ University of Science and Technology, Wuhan, China
}

The damage degree of neurons in perilesion at different time points was observed in order to explore the optimal operation occasion. Piglet lobar hematomas were produced by pressure-controlled infusions of $2.5 \mathrm{~mL}$ autonomous blood into the right frontal hemispheric white matter over $15 \mathrm{~min}$, and the metabolic changes were ambulatorily detected with MRS (MR spectroscopy) at 3rd, 12th, 24th and 48th $\mathrm{h}$ after hematoma induction. Brain tissues of perihematoma were also obtained at different time points. The transcription level of Bax gene was detected by in situ hybridization and apoptosis by TUNEL technique, and the pathologic change of neurons was observed under an electron microscope. The results showed that the number of Bax positive cells reached the peak at $24 \mathrm{~h}(79.00 \pm 4.243 / 5$ fields $)$. There was no significant difference in $A$ values between $3 \mathrm{~h}$ and $6 \mathrm{~h}, 12 \mathrm{~h}$ ( $p>0.05$ ), but there was significant difference between $24 \mathrm{~h}$ and $3 \mathrm{~h}$, $6 \mathrm{~h}, 12 \mathrm{~h}(\mathrm{p}<0.05)$. The number of apoptotic cells reached the peak at $24 \mathrm{~h}(p<0.001)$, and there was no significant difference between $3 \mathrm{~h}$ and $6 \mathrm{~h}(\mathrm{p}=0.999)$. The area of the apoptotic cells showed no significant difference between $3 \mathrm{~h}$ and $6 \mathrm{~h}$ or among $3 \mathrm{~h}, 6 \mathrm{~h}$ and $6 \mathrm{~h}$ $(\mathrm{p}>0.05$ ). Lac peak mainly occurred at $24 \mathrm{~h}$ and $48 \mathrm{~h}$, while on the healthy side, no Lac peak was detectable. The ratio of NAA/Cr $(\mathrm{N}-$ acetylaspartate/Creatine) presented a descent tendency, but there was no significant difference among the group before $12 \mathrm{~h}(\mathrm{p}>0.05)$, there was obviously significant difference between $3 \mathrm{~h}, 6 \mathrm{~h}$ and $24 \mathrm{~h}$, $48 \mathrm{~h}(\mathrm{p}<0.01)$. Under electronic microscopy, the neuronal damage surrounding hematoma in 3-6h was milder than in $24-48 \mathrm{~h}$. It was concluded that the secondary apoptosis, damage and metabolic disturbance of the neurons surrounding hematoma was milder in 3-6h in acute intracerebral hemorrhage, while obviously aggravated in $24-48 \mathrm{~h}$. An effective intervention is needed to reduce secondary damage as soon as possible. 